\title{
Association Between Clinical Value and Financial Cost of Cancer Treatments: A Cross-Sectional Analysis
}

\author{
Aaron P. Mitchell, MD, MPH${ }^{1}$; Sara M. Tabatabai, BS1; Pranammya Dey, BS1,2; Jennifer A. Ohn, MPH
} Michael A. Curry, MS'; and Peter B. Bach, MD, MAPP'

\begin{abstract}
Background: The cost of cancer treatment has increased significantly in recent decades, but it is unclear whether these costs have been associated with commensurate improvement in clinical value. This study aimed to assess the association between the cost of cancer treatment and 4 of the 5 NCCN Evidence Blocks (EB) measures of clinical value: efficacy of regimen/agent, safety of regimen/agent, quality of evidence, and consistency of evidence. Methods: This is a cross-sectional, observational study. We obtained NCCN EB ratings for all recommended, first-line, and/or maintenance treatments for the 30 most prevalent cancers in the United States and calculated direct pharmacologic treatment costs (drug acquisition, administration fees, guideline-concordant supportive care medications) using Medicare reimbursement rates in January 2019. We used generalized estimating equations to estimate the association between NCCN EB measures and treatment cost with clustering at the level of the treatment indication. Results: A total of 1,386 treatments were included. Among time-unlimited treatments (those administered on an ongoing basis without a predetermined stopping point), monthly cost was positively associated with efficacy $(\$ 3,036 ; 95 \% \mathrm{Cl}, \$ 1,782$ to $\$ 4,289)$ and quality of evidence $(\$ 1,509 ; 95 \% \mathrm{Cl}, \$ 171$ to $\$ 2,847)$ but negatively associated with safety $(-\$ 1,470 ; 95 \% \mathrm{Cl},-\$ 2,790$ to $-\$ 151)$ and consistency of evidence $(-\$ 2,003 ; 95 \% \mathrm{Cl},-\$ 3,420$ to $-\$ 586)$. Among time-limited treatments (those administered for a predetermined interval or number of cycles), no NCCN EB measure was significantly associated with treatment cost. Conclusions: An association between NCCN EB measures and treatment cost was inconsistent, and the magnitude of the association was small compared with the degree of cost variation among treatments with the same EB scores. The clinical value of cancer treatments does not seem to be a primary determinant of treatment cost.
\end{abstract}

J Natl Compr Canc Netw 2020;18(10):1349-1353 doi: $10.6004 /$ jnccn.2020.7574

\footnotetext{
${ }^{1}$ Health Outcomes Research Group, Department of Epidemiology and Biostatistics, Memorial Sloan Kettering Cancer Center, New York, New York; and ${ }^{2}$ Yale University School of Medicine, New Haven, Connecticut.
}

\section{Background}

Drug prices have become a significant problem in oncology. Initial list prices have increased at an exponential rate, ${ }^{1}$ and most new cancer drugs experience additional postmarketing price increases. ${ }^{2,3}$ These trends have resulted in sufficient financial toxicity. ${ }^{4}$

However, it is not clear whether newer, more expensive drugs have produced a clinical benefit commensurate with their financial burden. As prices have increased, the financial cost necessary to achieve the same survival improvement using newer drugs has also increased. ${ }^{5}$ In addition, a number of studies evaluating the prices of newly approved cancer drugs with respect to their clinical trial data have failed to find a positive relationship between the magnitude of improvements in patient outcomes and drug prices. ${ }^{6-13}$

Despite these observations, pharmaceutical industry representatives have long asserted that drug prices reflect underlying clinical value and are therefore justified. ${ }^{14-16}$ Whether increasing cancer drug prices reflect significant improvements in effectiveness is therefore germane to ongoing legislative attempts at drug price reforms.

Whether treatment price may reflect clinical benefit across all cancer treatment settings, not only among newly approved treatments for on-label indications as previously studied, has not been evaluated. In addition, the influence of recently approved immunotherapy drugs on the price-benefit association has not been evaluated. Therefore, we aimed to assess the correlation between the clinical value of cancer treatments and the cost of treatment, inclusive of all treatment regimens across all recommended uses, both approved and off-label.

\section{Methods}

NCCN publishes Evidence Blocks (EB), which assess cancer treatments on the following 5 measures using a

See JNCCN.org for supplemental online content. 
simple 1 to 5 scoring system: efficacy of regimen/agent (extent to which treatment improves survival and/or symptoms), safety of regimen/agent (likelihood of adverse effects, with fewer adverse effects receiving higher scores), quality of evidence (number and rigor of the supporting clinical trials), consistency of evidence (degree to which clinical trials agree on the degree of benefit), and affordability of regimen/agent (overall cost of treatment, with less expensive treatments receiving higher scores). ${ }^{17} \mathrm{~EB}$ scores reflect a synthesis of clinical evidence and expert opinion. The NCCN EB are the most comprehensive of the value frameworks developed in oncology, including all recommended treatments regardless of approval date or level of clinical evidence, and is the most widely recognized framework by oncologists. ${ }^{18}$

We extracted EB scores for all treatments used in the adjuvant, neoadjuvant, definitive treatment, first-line, or maintenance settings for the 30 highest-incidence cancers in the United States, current as of December 31, 2018. For each treatment, we calculated Medicare treatment costs, using the average sales price (ASP) file and Medicare Plan Finder prices, inclusive of administration and supportive care costs, as described in earlier research (for full methodologic details, see supplemental eAppendix 1, available with this article at JNCCN.org). ${ }^{19}$ Ancillary and nonmedical costs were not included. To avoid making misleading cost comparisons, we categorized each treatment as either "time-limited" (often, adjuvant or neoadjuvant treatments) or "time-unlimited" (often, treatments for advanced/metastatic disease). For timelimited treatments, we calculated costs across the full course of therapy; for time-unlimited treatments, we calculated the monthly cost of treatment.

To enable comparisons between treatments that were similar (eg, those that might be weighed against each other in a clinical treatment decision), we categorized all

\section{Table 1. Cost at Each Level of NCCN EB Measures}

\begin{tabular}{|c|c|c|c|c|c|c|}
\hline NCCN EB Measu & & \multicolumn{5}{|c|}{ EB Score } \\
\hline \multicolumn{7}{|c|}{ Time-unlimited $(n=541)$} \\
\hline \multirow[t]{3}{*}{ Efficacy } & Mean, USD & $\mathrm{N} / \mathrm{A}$ & 4,591 & 5,898 & 10,228 & 11,584 \\
\hline & Range, USD & N/A & $(4-43,078)$ & $(4-31,190)$ & $(6-64,630)$ & $(1,387-17,093)$ \\
\hline & $\mathrm{n}$ & 0 & 121 & 241 & 164 & 15 \\
\hline \multirow{2}{*}{ Safety } & Range, USD & $(8,050-8,050)$ & $(242-64,630)$ & $(16-43,079)$ & $(4-21,537)$ & N/A \\
\hline & $\mathrm{n}$ & 2 & 105 & 320 & 114 & 0 \\
\hline \multirow[t]{3}{*}{ Quality } & Mean, USD & 27,260 & 5,087 & 5,422 & 10,086 & 7,368 \\
\hline & Range, USD & $\mathrm{N} / \mathrm{A}$ & $(6-31,190)$ & $(4-43,079)$ & $(262-64,630)$ & $(6-19,119)$ \\
\hline & $\mathrm{n}$ & 1 & 76 & 251 & 178 & 35 \\
\hline \multicolumn{7}{|c|}{ Time-limited $(n=845)$} \\
\hline \multirow[t]{3}{*}{ Efficacy } & Mean, USD & N/A & 29,899 & 24,673 & 36,494 & 35,796 \\
\hline & Range, USD & N/A & $(498-294,412)$ & $(0-775,559)$ & $(0-500,982)$ & $(2,292-217,998)$ \\
\hline & $n$ & 0 & 75 & 347 & 359 & 64 \\
\hline \multirow[t]{3}{*}{ Safety } & Mean, USD & 33,607 & 32,846 & 27,475 & 44,140 & $N / A$ \\
\hline & Range, USD & $(1,637-149,110)$ & $(769-775,559)$ & $(0-487,859)$ & $(527-294,412)$ & N/A \\
\hline & $\mathrm{n}$ & 12 & 265 & 479 & 89 & 0 \\
\hline \multirow[t]{3}{*}{ Quality } & Mean, USD & 3,718 & 24,020 & 31,062 & 33,454 & 26,186 \\
\hline & Range, USD & $(2,584-4,853)$ & $(678-487,859)$ & $(0-500,982)$ & $(527-775,559)$ & $(1,637-116,677)$ \\
\hline & $\mathrm{n}$ & 2 & 94 & 416 & 315 & 18 \\
\hline
\end{tabular}

NCCN EB measures are scaled such that higher scores are always preferable.

Abbreviations: EB, Evidence Blocks; N/A, not applicable. 
treatments into treatment groups defined by (1) treatment indication, as defined by the NCCN EB; (2) timelimited versus time-unlimited; and (3) inclusion of radiation therapy and/or hematopoietic stem cell transfer as part of the treatment.

We used descriptive statistics to assess the distribution of treatment costs across NCCN EB scores separately for time-limited and time-unlimited treatments. We assessed the distribution for each measure separately and for the overall sum of efficacy, safety, quality of evidence, and consistency of evidence; affordability was omitted because it is a measure of treatment cost, which we measured separately.

We used generalized estimating equations, clustered at the level of the treatment group, to assess the independent association between treatment costs and each NCCN EB measure. Models were multivariable, using treatment cost as the dependent variable and the 4 included NCCN EB measures as independent variables. We conducted planned sensitivity analyses that included (1) general linear models without clustering, (2) omission of treatment groups containing $n=1$ treatment, (3) no supportive care costs, (4) more aggressive supportive care utilization, (5) modeling of logged treatment costs, (6) omission of treatment groups using radiation therapy or stem cell transfer, (7) rank-order testing (KruskalWallis test), and (8) treatment group-level fixed effects.

\section{Results}

The analysis included 1,386 treatments: 541 were timeunlimited and 845 were time-limited. For each NCCN EB measure, there was wide variation in treatment cost across each possible score. For example, for time-limited regimens that received an efficacy score of 5 (highest

A

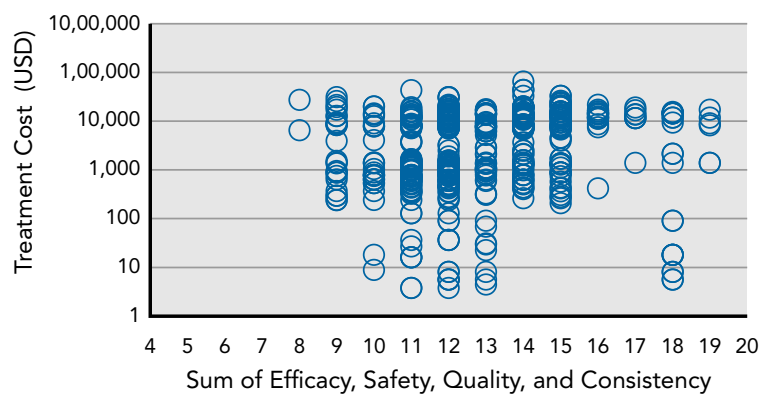

efficacy), the mean cost was $\$ 35,796$, with a range of $\$ 2,292$ to $\$ 217,998$ (Table 1).

Wide variation was also seen when comparing the cost of each treatment with its summary NCCN EB score (the sum of efficacy, safety, quality of evidence, and consistency of evidence) (Figure 1).

When we assessed the independent association between each NCCN EB measure and the cost for timeunlimited treatments, we found that efficacy was positively associated with treatment cost (estimated change in cost associated with 1-unit increase on efficacy measure; $\$ 3,036$; $95 \% \mathrm{CI}, \$ 1,782$ to $\$ 4,289$ ) as was quality of evidence $(\$ 1,509 ; 95 \% \mathrm{CI}, \$ 171$ to $\$ 2,847)$, whereas safety $(-\$ 1,470 ; 95 \% \mathrm{CI},-\$ 2,790$ to $-\$ 151)$ and consistency of evidence $(-\$ 2,003 ; 95 \% \mathrm{CI},-\$ 3,420$ to $-\$ 586)$ were negatively associated with cost (Table 2). Among time-limited treatments, no NCCN EB measure was significantly associated with cost.

Model estimates were largely consistent across sensitivity analyses (supplemental eTables 1-8). Among time-unlimited treatments, efficacy and quality of evidence were positively associated with cost and safety and consistency of evidence were negatively associated with cost; among time-limited treatments, NCCN EB measures were not associated with cost. A notable exception was fixed-effect modeling (supplemental eTable 8), which suggested a positive association between efficacy and cost and a negative association between quality of evidence and cost among time-limited treatments.

\section{Discussion}

This analysis suggests an association between the NCCN EB measures and treatment cost. However, in our primary analysis this association was present among only
B

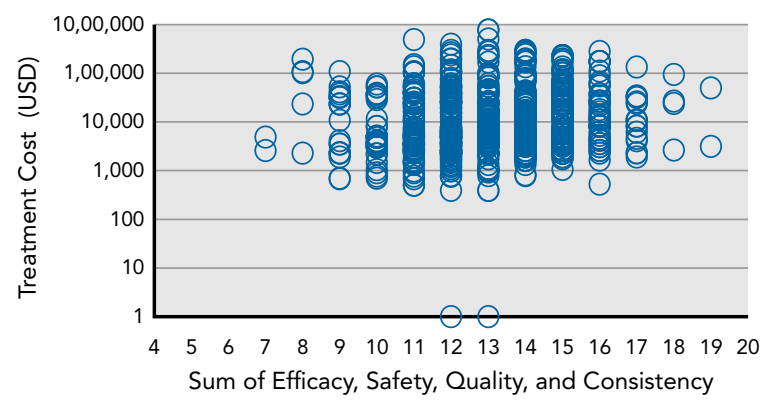

Figure 1. Distribution of treatment costs and NCCN EB scores. The $x$ axis represents the numeric sum of the efficacy, safety, quality of evidence, and consistency of evidence scores for each treatment. Because each measure ranges from 1 to 5 , the $x$ axis range is from 4 (lowest-clinical-value treatments) to 20 (highest-clinical-value treatments); NCCN EB measures are scaled such that higher scores are always preferable. (A) Timeunlimited treatments; cost represents cost per month of therapy $\left(\mathrm{N}=541\right.$; linear $\left.R^{2}=0.03\right)$. (B) Time-limited treatments; cost represents cost for full course of treatment $\left(N=845\right.$; linear $\left.R^{2}=0.004\right)$. For the 2 treatments with drug costs of $\$ 0$, we assigned a cost of $\$ 1$ to allow inclusion on a logarithmic scale.

Abbreviation: EB, Evidence Blocks. 


\begin{tabular}{|c|c|c|c|c|c|c|c|c|}
\hline \multirow[b]{2}{*}{ EB Measure } & \multicolumn{4}{|c|}{$\begin{array}{l}\text { Time-Unlimited } \\
\quad(n=541)\end{array}$} & \multicolumn{4}{|c|}{$\begin{array}{l}\text { Time-Limited } \\
\quad(n=845)\end{array}$} \\
\hline & Estimate & Lower $95 \% \mathrm{Cl}$ & Upper $95 \% \mathrm{Cl}$ & $P$ Value & Estimate & Lower $95 \% \mathrm{Cl}$ & Upper $95 \% \mathrm{Cl}$ & $P$ Value \\
\hline Efficacy, USD & 3,036 & 1,782 & 4,289 & $<.001$ & 5,248 & $-5,798$ & 16,293 & .35 \\
\hline Safety, USD & $-1,470$ & $-2,790$ & -151 & .03 & 1,964 & $-6,026$ & 9,953 & .63 \\
\hline Quality, USD & 1,509 & 171 & 2,847 & .03 & $-2,066$ & $-15,531$ & 11,399 & .76 \\
\hline Consistency, USD & $-2,003$ & $-3,420$ & -586 & .01 & 3,274 & $-7,954$ & 14,502 & .57 \\
\hline
\end{tabular}

Estimates represent the change in treatment cost associated with a 1-unit increase in the score on the indicated NCCN EB measure. NCCN EB measures are scaled such that higher scores are always preferable.

Abbreviation: EB, Evidence Blocks.

1 group of treatments (those intended to be administered until disease progression or unacceptable toxicity, designated herein as "time-unlimited") and explained little of the significant variation in treatment cost. Monthly treatment costs varied by tens of thousands of dollars within each level of the NCCN EB efficacy score; the estimated \$3,036 greater cost per month associated with a 1-unit increase in efficacy suggests that this measure accounts for little of the difference in cost between treatments. Implicitly, treatment costs are determined primarily by factors other than their clinical "value," as assessed by the NCCN EB measures.

The finding that some NCCN EB measures wereamong the time-unlimited treatments-inversely associated with cost is unexpected and intriguing. Because NCCN EB measures are scaled so that higher scores are always "better," this finding would imply that treatments tend to have higher prices when scoring worse on these measures. Because these associations were present on multivariable analysis, they would not be explained by a correlation between measures (eg, treatments with greater efficacy tended to have lower safety). Rather, all other things being equal, more-toxic treatments (for example) seem to be more costly. Because the inverse association between safety and cost was unaffected in the sensitivity analysis excluding supportive care costs, a greater need for supportive medications for more-toxic treatments also fails to explain this observation. The inverse associations between treatment cost and both Safety and consistency of evidence may support the hypothesis that treatment costs poorly reflect clinical value.

Sensitivity analyses were largely consistent with the primary analysis. One notable exception was the fixedeffect model, which found a positive association between efficacy and cost among both time-limited and time-unlimited treatments. This result suggests that such an association may be present, after accounting for the substantial intertreatment group variation in cost. However, the same model still failed to find an association with safety or consistency of evidence, and quality of evidence was negatively associated with cost.
This study has limitations related to the NCCN EB data source. Because the NCCN EB are determined through expert consensus there is some degree of subjectivity, which makes misclassification possible. Note also that in many cases in which we did not identify a statistically significant association between NCCN EB measures and costs, the wide confidence interval of our estimates indicated that our results would be consistent with potentially large associations.

\section{Conclusions}

To the extent that the associations identified in this study were inconsistent (evident in only 1 of the 2 treatment categories) and that the correlation between cost and clinical value was minimal (explaining little of the variation in cost), these findings suggest that the clinical value of treatments is not an important determinant of costs. However, our findings did suggest statistically significant associations between cost and some measures of clinical value; unexpectedly, some of these were "negative" associations, suggesting that lessvaluable drugs were priced higher. Therefore, to the extent that these associations do reflect a role of clinical value in determining treatment costs, our functional definition of "value" may prioritize some components of value (effectiveness of treatments, or efficacy) while failing to account for others (toxicity of treatment, or safety).

Submitted December 21, 2019; accepted for publication April 6, 2020

Author contributions: Study concept and design: All authors. Dataset creation: Mitchell, Tabatabai, Dey, Ohn. Data analysis: Mitchell, Curry. Results presentation: Mitchell, Tabatabai, Dey, Bach. Approval of final manuscript: All authors.

Disclosures: Dr. Mitchell has disclosed that he received a research award from Conquer Cancer Foundation, partially funded by Merck. Dr. Bach has disclosed that he has received personal fees from WebMD, Defined Health, JMP Securities, Mercer, Foundation Medicine, Grail, Morgan Stanley, Oppenheimer \& Co, Cello Health, Oncology Analytics, Anthem, Magellan Health, America's Health Insurance Plans, and EQRx; grants from Kaiser Permanente and Arnold Ventures; nonfinancial support from Oppenheimer \& Co, America's Health Insurance Plans, and Oncology Analytics; and other from Oncology Analytics. The remaining authors have disclosed that they 
have not received any financial consideration from any person or organization to support the preparation, analysis, results, or discussion of this article.
Correspondence: Aaron P. Mitchell, MD, MPH, Department of Epidemiology and Biostatistics, Memorial Sloan Kettering Cancer Center, 485 Lexington Avenue, New York, NY 10017. Email: mitchea2@mskcc.org

\section{References}

1. Bach P. Monthly and median costs of cancer drugs at the time of FDA approval 1965-2015. Memorial Sloan Kettering Cancer Center. Accessed December 9, 2015. Available at: https://www.mskcc.org/research-areas/ programs-centers/health-policy-outcomes/cost-drugs

2. Dusetzina SB, Huskamp HA, Keating NL. Specialty drug pricing and outof-pocket spending on orally administered anticancer drugs in Medicare Part D, 2010 to 2019. JAMA 2019;321:2025-2028.

3. Alevizakos M, Gaitanidis A, Appleman L. Quantification of the financial burden of antineoplastic agent price increases [abstract]. J Clin Oncol 2019;37(Suppl):Abstract 6519.

4. Zafar SY. Financial toxicity of cancer care: it's time to intervene. J Natl Cancer Inst 2015;108:djv370.

5. Howard DH, Bach PB, Berndt ER, et al. Pricing in the market for anticancer drugs. J Econ Perspect 2015;29:139-162.

6. Barnes TA, Amir E, Templeton AJ, et al. Efficacy, safety, tolerability and price of newly approved drugs in solid tumors. Cancer Treat Rev 2017;56: $1-7$.

7. Amir E, Seruga B, Martinez-Lopez J, et al. Oncogenic targets, magnitude of benefit, and market pricing of antineoplastic drugs. J Clin Oncol 2011; 29:2543-2549.

8. Mailankody S, Prasad V. Implications of proposed Medicare reforms to counteract high cancer drug prices. JAMA 2016;316:271-272.

9. Saluja R, Arciero VS, Cheng S, et al. Examining trends in cost and clinical benefit of novel anticancer drugs over time. J Oncol Pract 2018;14: e280-294.

10. Becker DJ, Lin D, Lee S, et al. Exploration of the ASCO and ESMO value frameworks for antineoplastic drugs. J Oncol Pract 2017;13:e653-665.
11. Vivot A, Jacot J, Zeitoun JD, et al. Clinical benefit, price and approval characteristics of FDA-approved new drugs for treating advanced solid cancer, 2000-2015. Ann Oncol 2017;28:1111-1116.

12. Del Paggio JC, Sullivan R, Schrag D, et al. Delivery of meaningful cancer care: a retrospective cohort study assessing cost and benefit with the ASCO and ESMO frameworks. Lancet Oncol 2017;18: 887-894.

13. Mailankody S, Prasad V. Five years of cancer drug approvals: innovation, efficacy, and costs. JAMA Oncol 2015;1:539-540.

14. Soriot P. Statement of Pascal Soriot, chief executive officer, AstraZeneca, before the Committee on Finance, United States Senate, concerning drug pricing in America: a prescription for change, part II. Accessed September 27, 2019. Available at: https://www.finance.senate.gov/imo/media/doc/ 26FEB2019SORIOT-ASTRAZENECA.pdf

15. Harris G. Cost of developing new medicine swelled to $\$ 802$ million, research study reports. The Wall Street Journal. December 3, 2001 Accessed September 27, 2019. Available at: https://www.wsj.com/ articles/SB1007336440403996240

16. Burkholder R. Pricing and value of cancer drugs. JAMA Oncol 2015;1: 841-842.

17. Carlson RW, Jonasch E. NCCN Evidence Blocks. J Natl Compr Canc Netw 2016;14(5 Suppl):616-619.

18. Shah-Manek B, Wong W, Ravelo A, et al. Oncologists' perceptions of drug affordability using NCCN Evidence Blocks: results from a national survey. J Manag Care Spec Pharm 2018;24:565-571.

19. Bach PB. Limits on Medicare's ability to control rising spending on cancer drugs. N Engl J Med 2009;360:626-633.

\section{See JNCCN.org for supplemental online content.}


Supplemental online content for:

\section{The Association Between Clinical Value and Financial Cost of Cancer Treatments: A Cross-Sectional Analysis}

Aaron P. Mitchell, MD, MPH; Sara M. Tabatabai, BS; Pranammya Dey, BS; Jennifer A. Ohn, MPH; Michael A. Curry, MS; and Peter B. Bach, MD, MAPP

J Natl Compr Canc Netw 2020;18(10):1349-1353

eAppendix 1: Supplementary Methods

eTable 1: Sensitivity Analysis 1: Association of NCCN Evidence Blocks Measures With Treatment Costs, Without Clustering at the Level of the Treatment Group

eTable 2: Sensitivity Analysis 2: Association of NCCN Evidence Blocks Measures With Treatment Costs, Treatment Groups With $\mathrm{N}=1$ Treatments Removed

eTable 3: Sensitivity Analysis 3: Association of NCCN Evidence Blocks Measures With Treatment Costs, Assuming No Supportive Care Costs

eTable 4: Sensitivity Analysis 4: Association of NCCN Evidence Blocks Measures With Treatment Costs, Assuming More Aggressive Supportive Care Utilization

eTable 5: Sensitivity Analysis 5: Association of NCCN Evidence Blocks Measures With Treatment Costs, Costs Modeled on Log Scale

eTable 6: Sensitivity Analysis 6: Association of NCCN Evidence Blocks Measures With Treatment Costs, Excluding Treatments That Involve Administration of Radiation or Stem Cell Transplantation

eTable 7: Sensitivity Analysis 7: Association of NCCN Evidence Blocks Measures With Treatment Cost, Using Rank-Ordered Kruskal-Wallis Testing

eTable 8: Sensitivity Analysis 8: Association of NCCN Evidence Blocks Measures With Treatment Costs, With Treatment Group Fixed Effects 


\section{eAppendix 1. Supplementary Methods}

\section{Treatment Group Categorization}

As we hypothesized that the NCCN Guidelines Panel members for different disease types might have different conceptions of what "expensive" and "inexpensive" treatments entail, we did not want to compare treatments either between cancer types or between different indications within the same cancer type. Therefore, we grouped treatments by indication, using the same indication groups identified in the NCCN Clinical Practice Guidelines in Oncology (NCCN Guidelines). Additionally, as our calculations included drug costs but not radiation therapy (RT) costs or costs associated with stem cell transplantation (SCT), we did not want to compare treatments that included these expensive treatments to those that did not. Therefore, we further grouped treatments with respect to the inclusion of RT and/or SCT. The resulting treatment groups included treatments that were for the same cancer indication, were the same with respect to being timelimited or time-unlimited, and which were also the same with respect to whether RT and/or SCT was part of the treatment.

If a regimen was described within the NCCN Guidelines as including RT as a component of therapy (eg., "cisplatin + RT"), we designated the regimen as including radiation. In cases where the regimen was described as occurring in the setting of planned radiation therapy (eg, "radiosensitizing chemotherapy to be given with RT"), we designated the regimen as not including radiation, under the presumption that in such cases the decision to treat with radiation may be made independently of the decision of whether to give pharmacologic therapy, or which pharmacologic agent to use.

To avoid making comparisons between treatments which are intended to be administered only for a limited duration of time (often, adjuvant or neo-adjuvant treatments) versus those which are intended to be administered for an unlimited duration of time (often, treatments for advanced/metastatic disease), which would not be meaningful comparisons of treatment cost, we categorized each treatment as either "time-limited" or "time-unlimited." For time-limited treatments, we determined the number of doses for the full treatment course, and calculated costs across the full intended course of therapy. For time-unlimited treatments, we calculated the average monthly cost of treatment. In some cases of timeunlimited treatments, some components of therapy were intended to be given for an unlimited duration while other components were given for only a shorter duration, or were given initially at higher doses before transitioning to a lower dose over the long term; in such cases, monthly treatment costs would be higher in the initial months of therapy than in later months. For example, for several treatments for metastatic, hormone-positive breast cancer, trastuzumab is administered in combination with cytotoxic drugs, at an initial dose of $8 \mathrm{mg} / \mathrm{kg}$ before transitioning to a lower dose of $6 \mathrm{mg} / \mathrm{kg}$ for future doses. In such cases, we calculated the number of all doses of all treatments that would be given during the first planned 12 months of treatment and calculated average monthly cost across these 12 months.

\section{Decisions Regarding Treatment Regimens and Dosing}

For each treatment, we identified the dose and the number of doses of each drug, based on the availability of sources in the following hierarchy: (1) dosing schedules specified within the NCCN Guidelines, (2) standard dosing levels specified on the drug label, (3) a dosing schedule from the reference or references cited by the NCCN Guidelines for each treatment, (4) a manual literature search in PubMed for a dosing schedule corresponding to the relevant indication. If an applicable dosing schedule was not attainable through any of these sources, the treatment was excluded.

If multiple dosing schedules for the same drug were included, we used the schedule with the least-frequent dosing interval and the lowest dose. If multiple combinations of different drugs and/or durations of therapy were provided (eg, " 4 to 6 cycles"), we selected the treatment and duration felt to best represent the current standard of care, as identified through discussion with clinical experts in the relevant cancer type if necessary.

To avoid inconsistencies in the prices paid by different insurers and because Medicare is the single largest payer for US cancer patients, ${ }^{1}$ we used Medicare prices in our analysis. For physician-administered drugs reimbursed under Medicare Part B, we used the January 2019 Medicare ASP file and identified the payment limit for the Healthcare Common Procedure Coding System (HCPCS) code corresponding to each drug. ${ }^{2}$ For oral drugs reimbursed under Medicare Part D, we used the Medicare Plan Finder website to identify the unit retail price, as previously described. ${ }^{3,4}$ We used drug doses corresponding to a 70 kilogram body weight, a $1.7 \mathrm{~m}^{2}$ body surface area, and normal renal function, where applicable.

In cases where the NCCN Guidelines named a class of drugs (eg, "aromatase inhibitor") rather than a specific drug, we used the cheapest option among those currently in common use in the United States for use in price calculations.

\section{Decisions Regarding Supportive Care Medications}

We determined which treatments warranted growth factor support by using the NCCN Guidelines for Myeloid Growth Factors. Not all regimens contained in the NCCN Guidelines for each cancer type are mentioned specifically in the 


\section{eAppendix 1. Supplementary Methods (cont.)}

myeloid growth factor guidelines. In such cases, where possible neutropenic fever risk was inferred from similar treatments. For example, if a drug was listed as having a high risk of neutropenic fever at a given dosing schedule, then we inferred that the risk of neutropenic fever would also be high for other treatments that differed by having a higher dose, a more frequent dosing schedule, and/or the addition of more drugs. In cases where such inference was not possible but risk of neutropenia was suspected based on the characteristics of the included drugs, we searched the clinical trial literature in PubMed to ascertain whether the treatment was associated with a high $(>20 \%)$ or intermediate $(10 \%-20 \%)$ risk of neutropenic fever. Growth factor treatment was assumed to occur once per cycle and occur in the form of peg-filgrastim.

We determined which treatments warranted antiemetic therapy by using the NCCN Antiemesis guidelines. For each treatment, the most highly-emetogenic agent was determined, and the number of administrations of that agent. We assumed that antiemetic treatment would be provided at the time of each administration of that agent. We included the antiemetic agents that are given routinely in the 2 to 3 days surrounding treatment for acute and delayed emesis prevention, but not oral agents taken at home on an as-needed basis.

We calculated treatment costs under two sets of assumptions: (1) assuming supportive care medications are given more aggressively and with higher-cost options, (2) assuming that they are given less aggressively and with lower-cost options. In the more-aggressive scenario, we assumed growth factor therapy was given for treatments at high risk and intermediate risk of neutropenic fever, and that of the available antiemetic options for the appropriate level of emetic risk, the option with the highest-cost and/or intravenous drugs was used. In the less-aggressive scenario, we assumed growth factor therapy was given for treatments at high risk of neutropenic fever, and that of the available antiemetic options, the option with the lowest-cost and/or orally-administered drugs was used. The less-aggressive scenario was our base-case assumption.

\section{Additional Decisions Regarding Dosing and Cost Calculations for Specific Cancer Types}

NSCLC: For initial cytotoxic therapy for advanced disease, treatment was assumed to last for 4 cycles where specified on the drug label (eg, carboplatin + pemetrexed + bevacizumab). For other cases where treatment could continue for 4 to 6 cycles (eg, carboplatin + pemetrexed), treatment was assumed to last for 6 cycles.

Breast: In cases where the drug class of aromatase inhibitors was recommended, anastrozole was identified as the lowest-cost agent and was used for price calculations.

Prostate: In cases where the drug class of first-generation anti-androgens was recommended, bicalutamide was used. In cases where the drug class of luteinizing hormone-releasing hormone (LHRH) agonists was recommended, leuprolide was identified as the lowest-cost agent and was used for price calculations. In cases where the drug class of LHRH antagonists was recommended, degarelix was used. For adjuvant treatment of node-positive or high-risk disease, two years of androgen deprivation therapy was assumed.

B-Cell Lymphomas: For hyper-CVAD regimens, the intrathecal chemotherapy was not included in cost calculations. For ibritumomab tiuxetan, normal platelet counts were assumed.

Colon and Rectal: 6 months was assumed as the standard duration of adjuvant therapy where not otherwise specified. Central Nervous System: For treatments including radiation therapy and concurrent + adjuvant temozolomide, we used dosing according to the protocol specified by Stupp et al. ${ }^{5}$ For anaplastic glioma treatments including radiation therapy and concurrent + adjuvant temozolomide, we used dosing according to the protocol specified by van den Bent et al. ${ }^{6}$ In cases where the drug class of somatostatin analogs was recommended, octreotide was used.

Bladder: For bladder-sparing protocols, we used dosing for patients who achieved complete response and avoided cystectomy.

Sarcoma: For gastrointestinal stromal tumor, adjuvant tyrosine kinase inhibitor therapy was assumed to be 3 years in duration.

Pancreatic: Dosing for single-agent infused 5-FU was inferred from common dosing regimens for colon cancer. For dosing of CapeOx regimen, we assumed an ECOG status of $0-1$.

Chronic Lymphocytic Leukemia: For regimens indicated for transformation to DLBCL, we inferred doing and treatment durations from the NCCN B-Cell Lymphoma Guidelines. We excluded regimens that contained alemtuzumab, because this drug is provided free of charge to patients with chronic lymphocytic leukemia by the manufacturer, and therefore list prices do not accurately reflect the cost of treatment. ${ }^{7}$

Melanoma: For dosing of talimogene laherparepvec, we used the dosing schedule reported by Andtbacka et al. ${ }^{8}$ We assumed patients did not experience clinically relevant disease progression, intolerability, complete remission, or lack of 


\section{eAppendix 1. Supplementary Methods (cont.)}

response during the first 12 months of treatment; we assumed that patients did not continue with the optional 6 additional months of treatment thereafter. We inferred melanoma lesion size from results reported by Chesney et al, ${ }^{9}$ which had a median sum of the products of the 2 longest perpendicular diameters (SPD) across all target lesions of $930 \mathrm{~mm}$; we assumed this SPD was accounted for by a single target lesion, which (assuming symmetry) would have diameters of $30.5 \mathrm{~mm}$ and $30.5 \mathrm{~mm}$. This would then translate into a largest diameter of $3.05 \mathrm{~cm}$, corresponding to a 2-mL dose per the drug label. For biochemotherapy, we assumed interferon was administered intravenously. For limb perfusion therapy, we calculated costs for the arm rather than the leg.

Myeloma: For regimens that included autologous stem cell transfer, we assumed an 8-week interval for this phase of therapy. For patients receiving carfilzomib + lenalidomide + dexamethasone prior to autologous stem cell transfer (ASCT), we assumed 9 cycles of treatment prior to ASCT consistent with protocol reported by Bringhen et al..$^{10}$ As there was no cited regimen for ixazomib + lenalidomide + dexamethasone, we extrapolated from dosing schedules for similar regimens.

Uterine: In cases where the drug class of "progestational agent" was recommended, progesterone was used. Duration of adjuvant therapy was assumed to be 3 cycles for intermediate risk and 6 cycles for high risk.

Gastric and Esophageal: Perioperative chemotherapy was assumed to use 3 cycles before and 3 cycles after surgery unless otherwise specified.

\section{Sample Cost Calculation}

Regimen: dose-dense AC followed by $\mathrm{T}+$ trastuzumab, for adjuvant or neoadjuvant therapy for HER2-positive breast cancer.

Regimen characteristics: Time-limited, high emetic risk, high risk of neutropenic fever

Doxorubicin cost $=4$ doses $* 60 \mathrm{mg} / \mathrm{m}^{2} * 1.7 \mathrm{~m}^{2} * \$ 0.3063 / \mathrm{mg}=\mathbf{\$ 1 2 4 . 9 7}$

Cyclophosphamide cost $=4$ doses $* 600 \mathrm{mg} / \mathrm{m}^{2} * 1.7 \mathrm{~m}^{2} * \$ 0.37822 / \mathrm{mg}=\mathbf{\$ 1 , 5 4 3 . 1 4}$

Paclitaxel cost $=4$ doses $* 175 \mathrm{mg} / \mathrm{m}^{2} * 1.7 \mathrm{~m}^{2} * \$ 0.154 / \mathrm{mg}=\$ \mathbf{1 8 3 . 2 6}$

Trastuzumab cost $=(1$ dose $* 4 \mathrm{mg} / \mathrm{kg} * 70 \mathrm{~kg} * \$ 10.6995 / \mathrm{mg})+(51$ doses $* 2 \mathrm{mg} / \mathrm{kg} * 70 \mathrm{~kg} * \$ 10.6995 / \mathrm{mg})=\$ 2995.86+$ $\$ 76,394.43=\$ \mathbf{7 9 , 3 9 0 . 2 9}$

Administration fees $=$ doxorubicin + cyclophosphamide + paclitaxel + trastuzumab $=$

$(4$ doses $* \$ 143.08)+(4$ doses $* \$ 143.08)+\left(4\right.$ doses $\left.^{*}[\$ 143.08+\$ 30.99+\$ 30.99]\right)+(52$ doses $* \$ 143.08)=\$ 572.32+\$ 572.32+$ $\$ 820.24+\$ 7440.16=\$ \mathbf{9}, \mathbf{4 0 5 . 0 4}$

Growth factor support $=4$ doses * $6 \mathrm{mg} * \$ 780.301 / \mathrm{mg}=\$ \mathbf{1 8 , 7 2 7 . 2 2}$

Antiemetic therapy $=4 *(1$ dose aprepitant $125 \mathrm{mg}$ orally +1 dose ondansetron $16 \mathrm{mg}$ orally +1 dose dexamethasone $12 \mathrm{mg}$ orally +3 doses dexamethasone $8 \mathrm{mg}$ orally +2 doses aprepitant $80 \mathrm{mg}$ orally) $=$

$4 *([1 * 125 \mathrm{mg} * \$ 1.1604 / \mathrm{mg})+(1 * 16 \mathrm{mg} * \$ 0.026 / \mathrm{mg})+(1 * 12 \mathrm{mg} * \$ 0.248)+(3 * 8 \mathrm{mg} * \$ 0.248)+(2 * 80 \mathrm{mg} * \$ 1.1604 / \mathrm{mg}])=$ $4 *(\$ 145.05+\$ 0.416+\$ 2.976+\$ 5.952+\$ 185.664)=4 * \$ 340.058=\$ \mathbf{1 , 3 6 0 . 2 3}$

Grand total $=\$ 124.97+\$ 1,543.14+\$ 183.26+\$ 79,390.29+\$ 9,405.04+\$ 18,727.22+\$ 1,360.23=\$ 110,734.15$

\section{References}

1. Evaluation of the oncology care model: performance period one. Accessed July 19, 2019. Available at: https://innovation.cms.gov/Files/reports/ocmsecondannualeval-pp1.pdf

2. Medicare Part B Drug Average Sales Price. Accessed January 1, 2019 Available at: https://www.cms.gov/Medicare/Medicare-Fee-for-ServicePart-B-Drugs/McrPartBDrugAvgSalesPrice/index.html

3. Bach PB. Limits on Medicare's ability to control rising spending on cancer drugs. N Engl J Med 2009;360:626-633.

4. Medicare Plan Finder. Accessed January 1, 2019. Available at: https://www. medicare.gov/find-a-plan/questions/home.aspx

5. Stupp R, Mason WP, van den Bent MJ, et al. Radiotherapy plus concomitant and adjuvant temozolomide for glioblastoma. N Engl J Med 2005;352: 987-996.

6. van den Bent MJ, Baumert B, Erridge SC, et al. Interim results from the CATNON trial (EORTC study 26053-22054) of treatment with concurrent and adjuvant temozolomide for $1 \mathrm{p} / 19 \mathrm{q}$ non-co-deleted anaplastic glioma: a phase 3, randomised, open-label intergroup study. Lancet 2017;390: 1645-1653.

7. Haas J. MS use leads to free Campath for leukemia patients. Accessed September 19, 2019. Available at: https://www.mdedge.com/clinicalneurologynews/article/55802/leukemia-myelodysplasia-transplantation/msuse-leads-free

8. Andtbacka RHI, Kaufman HL, Collichio F, et al. Talimogene laherparepvec improves durable response rate in patients with advanced melanoma. $\mathrm{J}$ Clin Oncol 2015;33:2780-2788.

9. Chesney J, Puzanov I, Collichio F, et al. Randomized, open-label phase II study evaluating the efficacy and safety of talimogene laherparepvec in combination with ipilimumab versus ipilimumab alone in patients with advanced, unresectable melanoma. J Clin Oncol 2018;36: 1658-1667.

10. Bringhen S, Petrucci MT, Larocca A, et al. Carfilzomib, cyclophosphamide, and dexamethasone in patients with newly diagnosed multiple myeloma: a multicenter, phase 2 study. Blood 2014;124:63-69. 


\begin{tabular}{|c|c|c|c|c|c|c|c|c|}
\hline \multirow[b]{2}{*}{ Evidence Blocks Measure } & \multicolumn{4}{|c|}{ Time-Unlimited $(\mathrm{N}=541)$} & \multicolumn{4}{|c|}{ Time-Limited $(\mathbf{N}=\mathbf{8 4 5})$} \\
\hline & Estimate & Lower $95 \% \mathrm{Cl}$ & Upper $95 \% \mathrm{Cl}$ & $P$ Value & Estimate & Lower $95 \% \mathrm{Cl}$ & Upper $95 \% \mathrm{Cl}$ & $P$ Value \\
\hline Efficacy, USD & 3,036 & 1,897 & 4,175 & $<.001$ & 5,248 & $-1,902$ & 12,397 & .15 \\
\hline Safety, USD & $-1,470$ & $-2,456$ & -484 & .004 & 1,964 & $-4,964$ & 8,891 & .58 \\
\hline Quality, USD & 1,509 & 12 & 3,006 & .05 & $-2,066$ & $-12,593$ & 8,461 & .70 \\
\hline Consistency, USD & $-2,003$ & $-3,685$ & -321 & .02 & 3,274 & $-8,181$ & 14,729 & .58 \\
\hline
\end{tabular}

Estimates represent the change in treatment cost associated with a one-unit increase in the score on the indicated Evidence Blocks measure. NCCN Evidence Blocks measures are scaled such that higher scores are always preferable.

\begin{tabular}{|c|c|c|c|c|c|c|c|c|}
\hline Evidence Blocks Measure & \multicolumn{4}{|c|}{ Time-Unlimited $(N=532)$} & \multicolumn{4}{|c|}{ Time-Limited $(\mathbf{N}=816)$} \\
\hline Efficacy, USD & 3,006 & 1,747 & 4,266 & $<.001$ & 6,811 & $-4,487$ & 18,109 & .24 \\
\hline Safety, USD & $-1,558$ & $-2,880$ & -235 & .02 & -97 & $-7,913$ & 7,719 & .98 \\
\hline Consistency, USD & $-1,838$ & $-3,274$ & -402 & .01 & 5,295 & $-5,945$ & 16,534 & 36 \\
\hline
\end{tabular}

Estimates represent the change in treatment cost associated with a one-unit increase in the score on the indicated Evidence Blocks measure. NCCN Evidence Blocks measures are scaled such that higher scores are always preferable.

\begin{tabular}{|c|c|c|c|c|c|c|c|c|}
\hline Evidence Blocks Measure & \multicolumn{4}{|c|}{ Time-Unlimited $(N=541)$} & \multicolumn{4}{|c|}{ Time-Limited $(\mathrm{N}=\mathbf{8 4 5})$} \\
\hline Efficacy, USD & 3,048 & 1,784 & 4,311 & $<.001$ & 3,806 & $-7,085$ & 14,697 & .49 \\
\hline Safety, USD & $-1,252$ & $-2,460$ & 159 & .09 & 4,726 & $-3,105$ & 12,556 & .23 \\
\hline Consistency, USD & $-2,162$ & $-3,567$ & -758 & .003 & 2,300 & $-8,950$ & 13,551 & .69 \\
\hline
\end{tabular}

Estimates represent the change in treatment cost associated with a one-unit increase in the score on the indicated Evidence Blocks measure. NCCN Evidence Blocks measures are scaled such that higher scores are always preferable. 


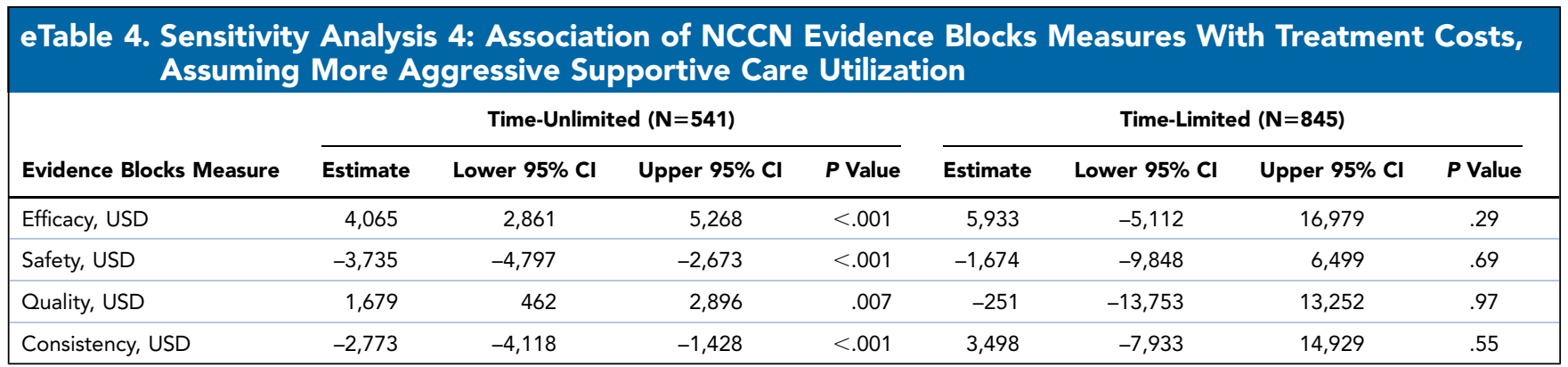

Estimates represent the change in treatment cost associated with a one-unit increase in the score on the indicated Evidence Blocks measure. NCCN Evidence Blocks measures are scaled such that higher scores are always preferable.

\begin{tabular}{|c|c|c|c|c|c|c|c|c|}
\hline Evidence Blocks Measure & \multicolumn{4}{|c|}{ Time-Unlimited ( $N=541)$} & \multicolumn{4}{|c|}{ Time-Limited ( $N=845$ ) } \\
\hline Efficacy, USD & 0.31 & 0.16 & 0.46 & $<.001$ & 0.11 & 0.01 & 0.21 & .03 \\
\hline Safety, USD & -0.39 & -0.59 & -0.19 & $<.001$ & -0.01 & -0.09 & 0.07 & .78 \\
\hline Consistency, USD & -0.2 & -0.38 & -0.02 & .03 & 0.01 & -0.12 & 0.13 & .90 \\
\hline
\end{tabular}

Estimates represent the change in treatment cost associated with a one-unit increase in the score on the indicated Evidence Blocks measure. NCCN Evidence Blocks measures are scaled such that higher scores are always preferable.

\begin{tabular}{|c|c|c|c|c|c|c|c|c|}
\hline Evidence Blocks Measure & \multicolumn{4}{|c|}{ Time-Unlimited $(\mathrm{N}=535)$} & \multicolumn{4}{|c|}{ Time-Limited ( $N=621)$} \\
\hline Efficacy, USD & 3,050 & 1,786 & 4,314 & $<.001$ & 8,459 & $-5,995$ & 22,914 & .25 \\
\hline Safety, USD & $-1,531$ & $-2,876$ & -186 & .03 & $-1,034$ & $-11,866$ & 9,799 & .85 \\
\hline Consistency, USD & $-2,005$ & $-3,423$ & -587 & .006 & 662 & $-13,299$ & 14,623 & .93 \\
\hline
\end{tabular}

Estimates represent the change in treatment cost associated with a one-unit increase in the score on the indicated Evidence Blocks measure. NCCN Evidence Blocks measures are scaled such that higher scores are always preferable. 


\begin{tabular}{|c|c|c|c|c|c|c|c|}
\hline & NCCN EB Measure & & \multicolumn{5}{|c|}{ EB Score } \\
\hline \multirow[t]{7}{*}{ Time-Unlimited $(\mathrm{N}=541)$} & \multirow[t]{2}{*}{ Efficacy } & Difference, USD & N/A & $-10,579$ & $-8,815$ & $-4,850$ & Ref \\
\hline & & $P$ value & N/A & $<.001$ & $<.001$ & .03 & $\mathrm{~N} / \mathrm{A}$ \\
\hline & \multirow[t]{3}{*}{ Safety } & Difference, USD & $-1,446$ & 2,292 & 2,264 & Ref & N/A \\
\hline & & $P$ value & .79 & .03 & .01 & N/A & N/A \\
\hline & & $\mathrm{N}$ & 2 & 105 & 320 & 114 & 0 \\
\hline & \multirow[t]{2}{*}{ Quality } & Difference, USD & 18,174 & $-2,485$ & $-1,385$ & 1,430 & Ref \\
\hline & & $P$ value & .02 & .38 & .59 & .55 & N/A \\
\hline \multirow[t]{9}{*}{ Time-Limited ( $N=845)$} & \multirow[t]{3}{*}{ Efficacy } & Difference, USD & N/A & $-7,945$ & $-13,502$ & $-2,087$ & Ref \\
\hline & & $P$ value & N/A & .55 & .20 & .83 & N/A \\
\hline & & $\mathrm{N}$ & 0 & 75 & 347 & 359 & 64 \\
\hline & \multirow[t]{3}{*}{ Safety } & Difference, USD & $-9,384$ & $-10,359$ & $-15,282$ & Ref & N/A \\
\hline & & $P$ value & .65 & .21 & .05 & N/A & N/A \\
\hline & & $\mathrm{N}$ & 12 & 265 & 479 & 89 & 0 \\
\hline & \multirow[t]{3}{*}{ Quality } & Difference, USD & $-11,086$ & 7,816 & 12,467 & 4,149 & Ref \\
\hline & & $P$ value & .83 & .75 & 59 & .85 & N/A \\
\hline & & $\mathrm{N}$ & 2 & 94 & 416 & 315 & 18 \\
\hline
\end{tabular}

Difference estimates represent the change in treatment cost associated with the indicated score of each Evidence Blocks measure, compared to the highest score received by any treatment on that measure as the referent. NCCN Evidence Blocks measures are scaled such that higher scores are always preferable. Abbreviations: EB, Evidence Blocks; N/A, not applicable.

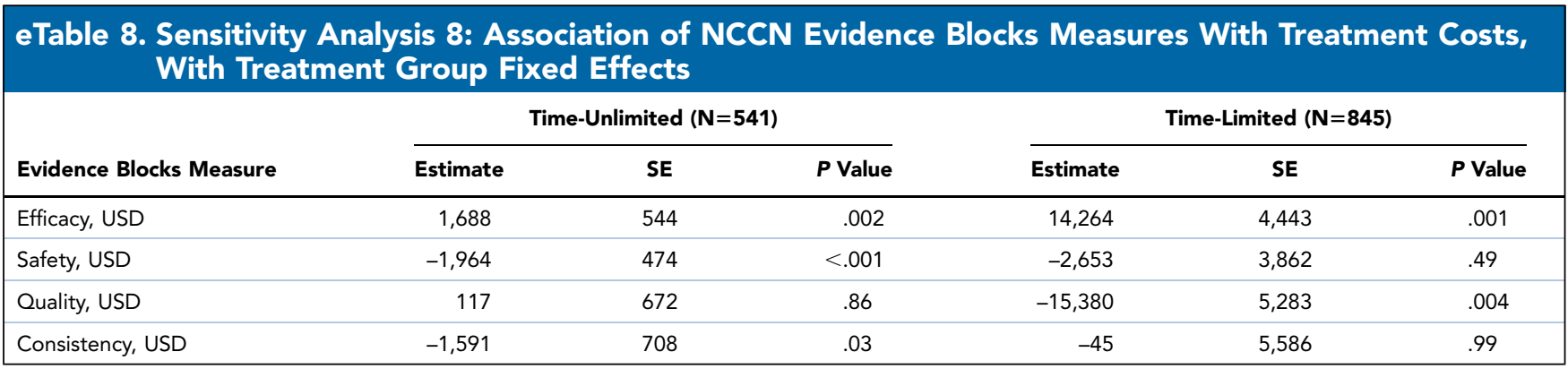

Estimates represent the change in treatment cost associated with a one-unit increase in the score on the indicated Evidence Blocks measure. NCCN Evidence Blocks measures are scaled such that higher scores are always preferable. 